\title{
Towards artificial cells for biomedical applications
}

\author{
Maria Wiesauer and Günther Knör* \\ Johannes Kepler University Linz, Institute of Inorganic Chemistry, Center for Nanobionics and Photochemical Sciences (CNPS), Altenberger Strasse 69, 4040 Linz, Austria
}

\begin{abstract}
A novel toolbox for future applications in precision nanomedicine and synthetic biology is the development of artificial cell organelles and the creation of cell-like structures operating similar to biological systems with a minimalistic set of building blocks. To achieve this ambitious goal, two major design strategies are followed in the field of synthetic biology. The top-down approach tries to generate a radically trimmed but still intact artificial cell by eliminating all non-essential components from the much more complex native systems. In contrast, bottom-up synthetic biology aims at constructing a functioning minimal cell by combining all essential building blocks step-by-step starting from scratch.

In the present contribution, the author's ongoing activities to develop artificial reaction centres for novel types of photoautotrophic processes and to provide fully biocompatible synthetic enzyme counterparts and artificial organelles as abiotic building blocks for bottom-up synthetic biology are summarized. This unique approach has the potential to create unprecedented minimal artificial cell-like systems that can be powered and readily controlled by light as an external stimulus, which will provide new perspectives for molecular photomedicine and photopharmacology.
\end{abstract}

\section{Introduction}

In current transdisciplinary research many efforts are made to better understand the boundary conditions and minimal requirements connected to the origin, distribution and sustain of living systems [1]. Scientists active in the field of synthetic biology [2] systematically explore the fundamental principles and limiting constraints existing for all organisms by creating non-native counterparts of biological systems. One of the ambitious goals of many researchers active in the field is trying to construct a primitive minimal version of a functioning artificial cell. This occurs either by top-down strategies, where apparently nonessential components of intact organisms are removed until a new kind of artificial cellular structure remains [3], or in a bottom-up fashion, where all required components are combined step-by-step [4]. These two complementary approaches are schematically illustrated in figure 1 .

Up to now, however, all claimed examples of top-down designed artificial cells still have to rely completely on the blueprints of naturally evolved structures and on the other hand no functioning cell-like constructs based on purely abiotic building blocks could be obtained with conventional bottom-up synthetic biology strategies. In the following sections, an alternative and now gradually maturing approach to achieve a minimal artificial cell based on light-controlled building blocks is briefly introduced.

\section{Photochemical control of intracellular functions}

\section{Light-mediated energy supply and information flow}

Life requires an orchestrated flow of energy, matter and information. Photons are ideally suited to target for the construction of a minimal cell-like structure, since they can selectively interact with matter as the fundamental carriers of energy and information with an excellent spatial and temporal resolution at the speed of light [5-7]. Such an approach has been systematically elaborated in the last decades $[5,6]$ and now allows to switch on and off the desired functions inside both native and artificial cells on demand [7]. At the same time, the application of synthetic building blocks for the photochemical control of intracellular functions represents the most straightforward strategy for creating independent photoautotrophic systems including artificial photosynthetic reaction centers [8] that can be directly supplied with solar radiation as their only energy source for metabolism (Figure 2).

\section{Light-triggered metabolic pathways}

One of the logical expansions of this versatile concept requires the control of photocatalytic one-pot multistep reactions using different excitation wavelengths or other orthogonal tools to address a certain set of photocatalysts for driving vectorial substrate conversion cascades resembling natural metabolic pathways. Once the functioning lightcontrolled components replacing native biological key-steps have been designed, their coupling to cascade processes in a metabolismlike fashion indeed also becomes possible [7]. Controlled and switched on and off by means of light as the external stimulus, this stepwise biomimetic transformation of substrates and metabolites $(\mathrm{A} \rightarrow \mathrm{B} \rightarrow \mathrm{C} \rightarrow \ldots)$ can occur in a one-pot version in the same celllike compartment [7] or could also be driven across self-assembled sub-structures separated by permeable membranes as schematically depicted in figure 3.

In the context of artificial enzyme function, meanwhile, the rational application of photochemical key-steps in such biomimetic catalytic systems has been systematically elaborated in depth, and it could be

*Correspondence to: Günther Knör, Johannes Kepler University Linz, Institute of Inorganic Chemistry, Center for Nanobionics and Photochemical Sciences (CNPS), Altenberger Strasse 69, 4040 Linz, Austria, Tel: +43(0)732-2468-5100; E-mail: guenther.knoer@jku.at

Key words: nanobionics, photomedicine, artificial enzymes, light-controlled metabolism, bottom-up synthetic biology, abiotic cells, synthetic organelles, chemical-biological hybrid systems, optogenetics, photopharmacology

Received: July 31, 2019; Accepted: August 16, 2019; Published: August 19, 2019 
shown that competitive or even superior functional counterparts of native oxidoreductase enzymes, nucleases and even more complex multienzyme reaction centers are readily obtained with this new strategy based on photo-assisted key-steps and full spatio-temporal light-control of catalytic performance [7]. Regarding the bioenergetic aspects of this approach, the first fully functional counterpart of the complete energy-trapping and solar-to-fuel conversion cascade otherwise only feasible with the natural photosystem I of green plants has been reported [8].
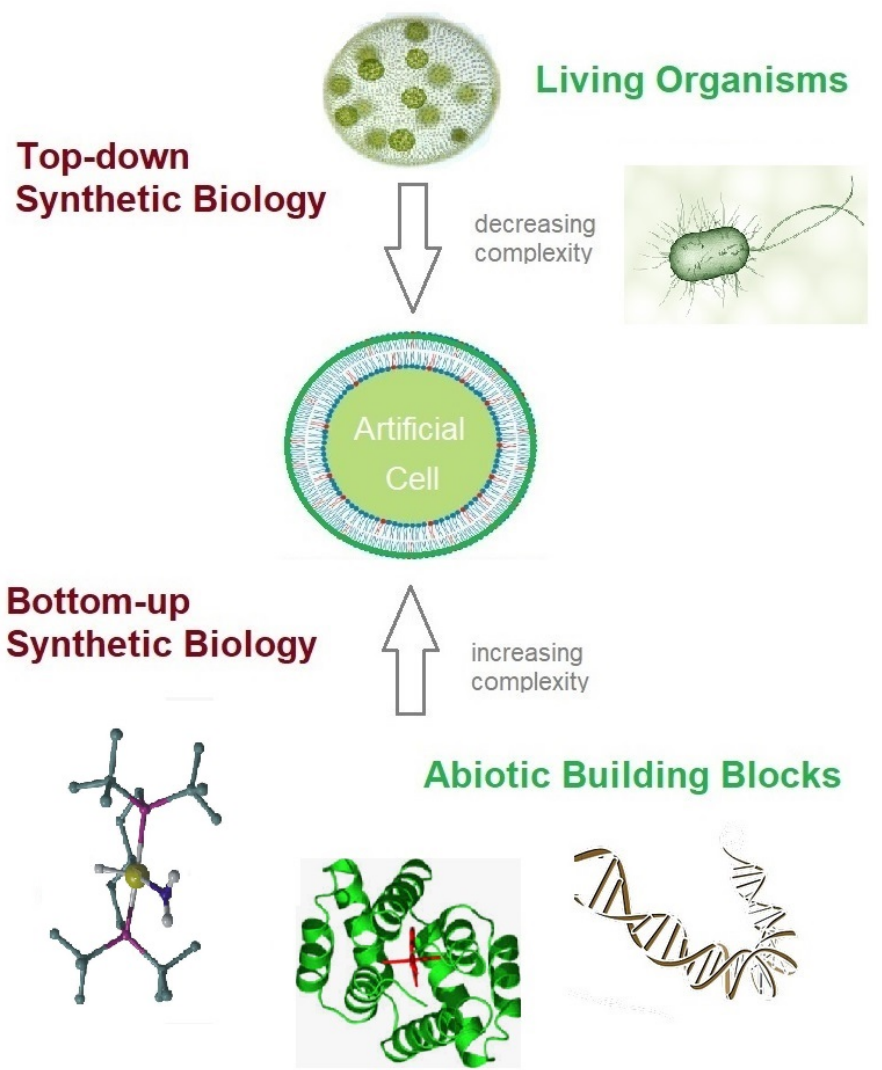

Figure 1. Comparison of the two different strategies followed in current synthetic biology research with the common goal of generating a minimal version of an artificial cell (center). Elimination of all non-essential components from native cells in order to reduce the level of complexity of living systems represents the so-called "top-down" approaches (top). The "bottom-up" approach aims at the creation of artificial cell-like system by bringing together a minimal set of functional synthetic building blocks (bottom).

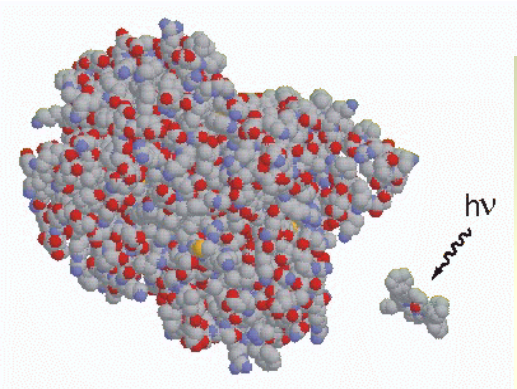

Native Enzyme

Photozyme

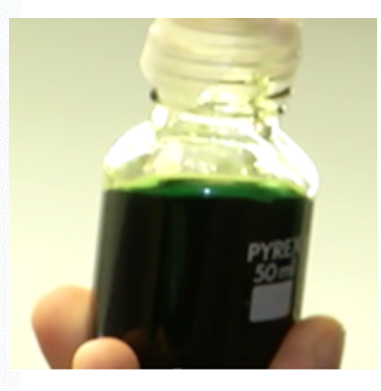

Figure 2. Schematic representation of the strategy to substitute the functional role of proteinand nucleic acid-based native counterparts by low-molecular-weight light-responsive keycomponents for creating minimalistic versions of photochemically controlled artificial organelles and cells [5-7].

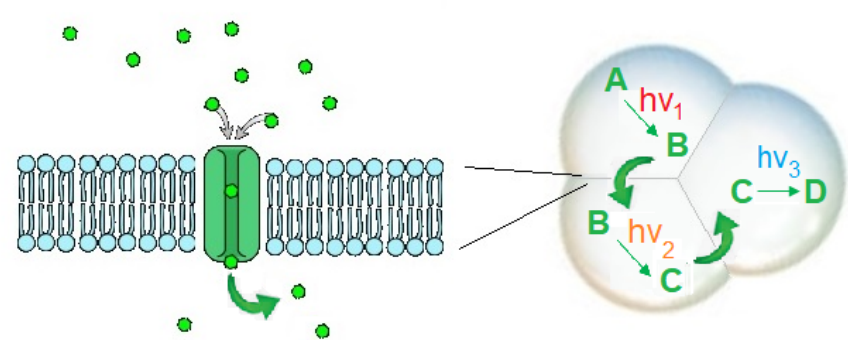

Figure 3. Stepwise metabolism-like substrate conversion fully controlled by light excitation using different wavelength and intensity as an external regulation mechanism. The individual photocatalytic steps are carried out in self-assembled vesicle structure with membrane pores allowing an exchange of the metabolites similar to the model systems introduced by Elani et al. [9]
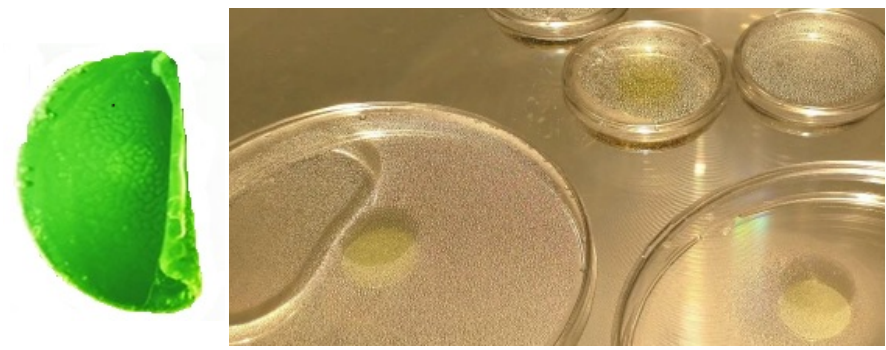

Figure 4. Photoenzyme model compounds and artificial photosynthetic reaction centres from the author's lab tested for their performance in cell and tissue-like hydrogel environments.

Another step forward to mimicking natural systems of higher complexity is the incorporation of artificial photoenzymes into chemical environments such as artificial cell-like structures, tissuelike environments or synthetic organelles to provide defined threedimensional architectures with a given light-controllable function. First steps in this direction are discussed in the next section below.

\section{Carrier matrices for artificial organelles and cells}

In nature all living cells consist of a cell membrane enclosing the water-based cytoplasma carrying a plethora of different functional components and metabolites. In larger biological tissues, ensembles of many similar cells are connected to achieve their specific functions in a synergetic way. The design of a primitive synthetic carrier material for artificial cells could thus include the shape-defining properties of an abiotic tissue matrix permeated by the cytosol as the reaction medium and containing cell-like compartments and embedded or attached artificial organelles as the functional subunits.

Soft and optically transparent hydrogel materials represent a straightforward option for the verification of such an artificial threedimensional tissue matrix for bottom-up synthetic biology applications due to their excellent biocompatibility, high water content and wellestablished usefulness in the fields of life-sciences and medicine (Figure 4).

Although these latter aspects are currently still in their infancy and a functioning artificial cell is not in sight today, a bright future can be expected for the application of light-control and photochemical keysteps in the field of bottom-up biology.

\section{Acknowledgement}

Support by the Austrian Science Fund (FWF) Project number W 1250 "DK NanoCell" and DK9: "Photochemical Control of Cellular Functions") is gratefully acknowledged. 


\section{References}

1. Kitadai N, Maruyama S (2018) Origins of Building Blocks of Life: A Review. Geosci Frontiers 9: 1117-1153.

2. Benner SA, Sismour AM (2005) Synthetic biology. Nat Rev Genet 6: 533-543. [Crossref]

3. Hutchison CA 3rd, Chuang RY, Noskov VN, Assad-Garcia N, Deerinck TJ, et al. (2016) Design and synthesis of a minimal bacterial genome. Science 351: aad6253. [Crossref]

4. [No authors listed] (2018) Bottom-up biology. Nature 563: 171. [Crossref]

5. Knör G (2001) Bionic Catalyst Design: A Photochemical Approach to Artificial Enzyme Function. Chem Bio Chem 7: 593-596. [Crossref]
6. Knör G (2009) Artificial Enzyme Catalysis Controlled and Driven by Light. Chem Eur $J$ 15: 568-578.

7. Knör G (2016) The Concept of Photochemical Enzyme Models - State of the Art. Coord Chem Rev 325: 102-115.

8. Knör G (2015) Recent Progress in Homogeneous Multielectron Transfer Photocatalysis and Arti-ficial Photosynthetic Solar Energy Conversion. Coord Chem Rev 304-305: 102-108.

9. Elani Y, Trantidou T, Wylie D, Dekker L, Polizzi K, et al. (2018) Constructing Vesiclebased Artificial Cells with Embedded Living Cells as Organelle-like Modules. Scienti Reports 8: 4564

Copyright: (C2019 Wiesauer M. This is an open-access article distributed under the terms of the Creative Commons Attribution License, which permits unrestricted use, distribution, and reproduction in any medium, provided the original author and source are credited. 